\title{
20. NEOGENE MULTISPECIES PLANKTONIC FORAMINIFER STABLE ISOTOPE RECORD, SITE 871, LIMALOK GUYOT ${ }^{1}$
}

\author{
Paul N. Pearson ${ }^{2}$ and Nicholas J. Shackleton ${ }^{3}$
}

\begin{abstract}
Carbon $\left(\delta^{13} \mathrm{C}\right)$ and oxygen $\left(\delta^{18} \mathrm{O}\right)$ stable isotope measurements were made on over 30 species of planktonic foraminifers from the lower Miocene to Pleistocene interval of the pelagic cap on Limalok Guyot (ODP Site 871), a low-latitude site in the western Pacific. Two reference taxa, Globigerinoides ruber and "Globoquadrina" venezuelana, show a consistent relative offset in $\delta^{13} \mathrm{C}$ and $\delta^{18} \mathrm{O}$ indicative of shallow- and deep-water habitats, respectively. Globigerinoides ruber exhibits an apparent carbon isotope vital effect of $-0.5 \%$ throughout the Neogene record. By comparing them to $G$. ruber and " $G$." venezuelana, other taxa are also interpreted in terms of their preferred classification depth, broadly confirming previously reported depth rankings. Many species show possible carbon isotope vital effects, including Globigerinella praesiphonifera, Fohsella spp., and Paragloborotalia mayeri. The isotopic record of "Dentglobigerina" altispira, which has usually been interpreted as a shallow-water dweller, bears little similarity to that of $G$. ruber, and it is speculated that it may be a relatively deep-dwelling species with a strong vital effect in both $\delta^{18} \mathrm{O}$ and $\delta^{13} \mathrm{C}$. Contrary to recent studies, no evidence for a change in depth habitat in the evolution of Fohsella was found. Similarly, the Globigerinoides bisphericus-Praeorbulina-Orbulina lineage apparently evolved without a change in depth habitat.

Anomalously high paleotemperatures for an extended period in the early to middle Miocene are interpreted as recording the existence of a locally restricted warm current over the site. Oxygen isotope data for this period are the most negative so far reported. Long-term trends in the carbon isotope record probably reflect global trends in organic carbon burial. For example, the midMiocene carbon isotope "Monterey" excursion is well reflected in the data.
\end{abstract}

\section{INTRODUCTION}

A growing mass of data is accumulating in the literature for multispecies stable isotopic studies of Neogene planktonic foraminifers (e.g., Douglas and Savin, 1978; Keller, 1985; Vincent et al., 1985; and other studies in Kennett, 1985; Gasperi and Kennett, 1992, 1993a, 1993b; Norris et al., 1993, 1994; Hodell and Vayavananda, 1994). These studies have amply confirmed that both carbon $\left(\delta^{13} \mathrm{C}\right)$ and oxygen $\left(\delta^{18} \mathrm{O}\right)$ stable isotopic ratios consistently differ between species in an assemblage and that the broad isotopic signature of a species tends to be a conservative feature over long periods of time and is comparable from site to site. The characteristic isotopic ratios of a species are thought to retain information of its life environment. Because different species have different environmental preferences, interspecies differences reflect environmental gradients at a site, provided certain assumptions prove justified. For example, inferences can be made on the degree of seasonality, surface- and intermediate-water temperatures, the strength and stability of the thermocline, levels of primary productivity, and the degree of vertical water-mass stratification (see Pearson et al. [1993] for a recent discussion). Such multispecies isotope studies, especially comparisons in space and time, potentially provide valuable insights in paleoceanographic study.

Multispecies isotope data are also of interest with respect to evolutionary patterns in planktonic foraminifers. Clearly, some species must have changed their life habitat at times in the past to produce the variety we see today. Fundamental questions include the following: Were these habitat changes gradual or sudden? Were they synchronous across and between ocean basins? Were they accompanied by morphological (anagenetic) evolution? Is biological speciation (cladogenesis) generally associated with habitat shift? The excellent fossil record of

\footnotetext{
${ }^{1}$ Haggerty, J.A., Premoli Silva, I., Rack, F., and McNutt, M.K. (Eds.), 1995. Proc ODP, Sci. Results, 144: College Station, TX (Ocean Drilling Program).

${ }_{2}^{2}$ Department of Earth Sciences, University of Cambridge, Downing Street, Cambridge. CB2 3EQ, United Kingdom. (Present address: Department of Geology, Wills Memoria Building, University of Bristol, Queens Road, Bristol BS8 IRJ, United Kingdom.)

${ }^{3}$ Godwin Laboratory for Quaternary Research, University of Cambridge. Free School Lane, Cambridge, CB2 3RS, United Kingdom.
}

planktonic foraminifers, in combination with the environmental information preserved by their isotopic ratios, surely provides the paleobiologist's best opportunity to examine aspects of the relationship between evolutionary pattern and environmental change. The nature of this relationship has been described as one of the remaining unanswered questions of evolutionary theory (Maynard Smith, 1988).

The potential in this approach is very great and only just beginning to be exploited. For example, in parallel studies published recently, Norris et al. (1993) and Hodell and Vayavananda (1994) found that the Fohsella lineage of the middle Miocene shifted its $\delta^{18} \mathrm{O}$ signal at about the time the first fully "keeled" individuals appeared. In both studies, "Dentoglobigerina" altispira was used as a reference for shallow-water conditions. The inference drawn from these studies was that the evolution of a keel in Fohsella was closely associated with a migration into a deeper habitat. On the other hand, Norris et al. (1994) showed that morphological evolution in the mid-latitude Globoconella lineage, including the acquisition of a keel, was not accompanied by an isotopic change.

Clearly, it is desirable to add to the number of lineages that have been studied so as to estimate the range and frequency of isotopic/ evolutionary pattern shown by planktonic foraminifers. The more interesting examples, such as Fohsella, should be studied at a variety of sites to identify potential geographical patterns. Furthermore, a wide range of species should be examined to provide a reference frame. And most fundamentally, the rationale for assigning depth habitats to extinct species by their isotopic signal should continually be scrutinized in the light of new data. Then, as the habitat evolution of many species becomes better understood, a more informed choice can be made in selecting taxa for monitoring paleoceanographic conditions in different environments.

\section{SITE 871}

Site 871 was drilled through the central and thickest part of the pelagic cap on Limalok Guyot, a seamount toward the southern end of the Ratak Chain in the Marshall Islands group (see site map preceding title page). Preliminary investigations at this site are described in Premoli Silva, Haggerty, Rack, et al. (1993). The pelagic sediments at 
this site were recovered using the advanced hydraulic piston corer (APC) in two adjacent holes (871 A and 871B). The most complete recovery was achieved in Hole $871 \mathrm{~A}$, and all the data in this study were measured on samples from that hole. The planktonic foraminifer biostratigraphy of Site 871 is described in Pearson (this volume).

The pelagic sediments that accumulated on the guyot top differ from sediments found in other environments. The lithology in Hole $871 \mathrm{~A}$ is very soft and unconsolidated foraminifer and nannofossilforaminifer ooze. In general, very little of the bulk sediment is in the fine fraction range $(<150 \mu \mathrm{m})$, as a result of winnowing by bottom currents. Fragmentation of tests is common, and it is severe in some samples. Reworking and hiatuses are also common features and follow from the high-energy conditions on the guyot top. Mixing of the "soupy" unconsolidated sediment in the core liners was often observed on recovery, and sporadic severe downhole contamination caused by caving at the top of the hole was unavoidable. For these reasons, the recovered section is not suitable for high-resolution paleoceanographic study.

On the other hand, the chemical state of preservation in much of the material is exceptionally good. Scanning electron microscopy was conducted on at least one sample from every core (see Pearson, this volume; Israelson et al., this volume). Despite the common fragmentation, other evidence of dissolution, such as surface etching, is sparse. No significant crystalline overgrowths were observed either on the inside or outside of tests. These observations lend confidence that the original isotopic ratios of the calcite at the time of crystallization are preserved.

In this study, a relatively long time interval was studied at a low resolution. This strategy plays to the strengths of the section, as foraminifer tests are well preserved down to the base of the pelagic cap, which is of early Miocene age (corresponding to planktonic foraminifer Zone N4/5). Thus, in addition to the isotopic characterization of long-term evolutionary transitions in the fauna, the broad paleoceanographic history of the site can be studied.

\section{METHODS}

Sediment samples of $10 \mathrm{~cm}^{3}$ volume were washed, wet sieved on a $150-\mu \mathrm{m}$ mesh, and dried at $50^{\circ} \mathrm{C}$. Each sample analyzed here consists of multiple picked specimens of the same species, commonly $10-15$ individuals. This procedure has the advantage that time-series data are less "noisy" and closer to the species mean value than are measurements made on individual specimens. Information on intraspecific variation is lost, however. All measurements were made on a VG Prism mass spectrometer at the Godwin Laboratory, University of Cambridge, and are expressed relative to the PDB standard.

Different sampling frequencies were adopted for different species. Sampling was concentrated on "marker species" characteristic of different environments (e.g., "Globoquadrina" venezuelana, Globigerinoides ruber, see below) and lineages of particular evolutionary or taxonomic interest (e.g., Fohsella, Praeorbulina, Globigerinatella). The results of each species or species-group are discussed in turn below. Aspects of the paleoceanography of Site 871 are then discussed in the light of the multispecies data.

\section{INFERRING DEPTH HABITAT}

A detailed review and discussion of the standard reasoning for inferring planktonic foraminifer depth habitats from isotopic data can be found in Pearson et al. (1993). Briefly, a shallow-dwelling species should secrete calcite with a relatively negative $\delta^{18} \mathrm{O}$ because of the warm temperature of surface waters. In open-ocean sites, a large change in temperature, and hence $\delta^{18} \mathrm{O}$ of foraminifer calcite, occurs with depth. Ideally, carbon isotopes are also depth related. Dissolved $\mathrm{CO}_{2}$ in surface waters tends to be more positive with respect to $\delta^{13} \mathrm{C}$ than deep waters because biological productivity, which preferentially removes ${ }^{12} \mathrm{C}$, is restricted to the photic zone. Thus, the $\delta^{13} \mathrm{C}$ and $\delta^{18} \mathrm{O}$ of a range of species living at different depths should be inversely correlated.
However, it is known that some species exhibit a consistent offset from expected equilibrium isotopic values as a result of so-called "vital effects." In general, offsets in $\delta^{18} \mathrm{O}$ that have been reported for living species are mostly small $(<1.0 \%)$ and negative (e.g., Berger, 1969; Kahn, 1979; Bouvier-Soumagnac et al., 1986; Sautter and Thunell, 1991; Spero, 1992), whereas for $\delta^{13} \mathrm{C}$ the effect may be relatively large $(>1.0 \%)$ and either negative (Kahn, 1979; Erez and Honjo, 1981) or positive (Spero and Williams, 1988; Spero, 1992). A species-specific vital effect will clearly give a misleading result in a depth ranking. However, in a limited way, it is possible to identify suspected vital effects by discordance in the oxygen and carbon isotopic rankings, or by parallelism in the data of an unknown species to the record of a reference species.

\section{LINEAGE ISOTOPIC CHARACTERISTICS}

All isotope data measured as part of this study are given in Table 1, and the entire data set is plotted in Figure 1. The shallow- and deepwater reference taxa are highlighted in the figure, as are two additional species with unusual ratios, Globigerinella praesiphonifera (crosses) and Clavatorella bermudezi (a single sample, diamond), which are discussed below. Other species are left undifferentiated so as not to confuse the figure. In general, the data obtained in this study support the general picture of planktonic foraminifer depth stratification reviewed by Keller (1985) and Corfield and Cartlidge (1991). The important lineages analyzed in this study are discussed in turn below.

\section{Globigerinoides ruber and Globigerinoides trilobus}

Both of the shallow-water reference taxa selected in this study belong to the genus Globigerinoides. Globigerinoides ruber and G. trilobus are extant species, although it is possible that Miocene " $G$. ruber" is a related homeomorph of the modern species and should properly be called G. subquadratus (see taxonomic discussion in Pearson, this volume). Plankton tow studies (e.g., Fairbanks et al., 1982; Ravelo and Fairbanks, 1992) show that both G. ruber and G. trilobus preferentially inhabit the surficial mixed layer and so record surface-water conditions. However, modern adult $G$. ruber typically records a vital effect offset of about $-0.5 \%$ with respect to the $\delta^{13} \mathrm{C}$ of dissolved $\Sigma \mathrm{CO}_{2}$ in the seawater from which it calcifies (Fairbanks et al., 1982).

It can be seen from Figure 1 that Globigerinoides spp. record the most negative $\delta^{18} \mathrm{O}$ in almost all assemblages throughout the Neogene, but that $G$. ruber is often not the most positive with respect to $\delta^{13} \mathrm{C}$. This is particularly evident in samples in which Globigerinoides bisphericus or the Praeorbulina-Orbulina lineage were picked (especially between 60 and $90 \mathrm{mbsf}$ ). It appears from these data that $G$. ruber has exhibited a fairly consistent carbon isotope vital effect of approximately $-0.5 \%$ since the late early Miocene at this site. This observation is circumstantial evidence for the genetic unity of modern G. ruber and the Miocene "G. subquadratus" of some authors.

\section{"Globoquadrina" venezuelana}

All modern "deep-dwelling" taxa are thought to spend part of their life cycle in the surface mixed layer (Hemleben et al. 1989). However, those that calcify much of their test in the lower thermocline or below record a "deeper" signal than those in the mixed layer. In this study, "Globoquadrina" venezuelana was the most commonly picked inferred deep-dwelling species and, as expected, consistently recorded the most positive $\delta^{18} \mathrm{O}$ and among the most negative $\delta^{13} \mathrm{C}$ of any species during the Miocene (with the exception of Globigerinella praesiphonifera, discussed below). Other studies have commonly used " $G$." venezuelana as a deep-dwelling reference taxon (e.g., Barrera et al., 1985; Gasperi and Kennett, 1993b). Although " $G$." venezuelana is extinct, there is no reason in the data presented here to question the generally accepted view of its depth ecology. 


\section{Globigerinella}

Globigerinella siphonifera ( $G$. aequilateralis of some authors) is an extant symbiotic species that is often found living at a relatively shallow level, but somewhat deeper than other shallow-dwelling forms (Fairbanks et al., 1982). The isotopic results obtained here are consistent with that habitat although the directly ancestral morphospecies, $G$. praesiphonifera of the Miocene, gives unexpected results. Data from G. praesiphonifera are shown as crosses in Figure 1 and are compared to the records of Globigerinoides ruber and "Globoquadrina" venezuelana for an interval in the lower to middle Miocene in Figures $2 \mathrm{~A}$ and $2 \mathrm{~B}$. The $\delta^{13} \mathrm{C}$ of " $G$." praesiphonifera is relatively negative, considerably more so than $G$. venezuelana, and much more than would be expected from its $\delta^{18} \mathrm{O}$, which generally records values intermediate between shallow- and deep-water reference taxa. The carbon isotope offset is interpreted as a probable vital effect. Note that Kahn (1979) recorded a large negative vital effect offset for $G$. siphonifera, although the mechanism for such an effect is not clear. Note that the $\delta^{13} \mathrm{C}$ record of the shallow-water dweller $G$. ruber is roughly parallel to the record of $G$. praesiphonifera, which also supports the hypothesis of a large and consistent vital effect.

\section{"Dentoglobigerina" altispira}

A species that is commonly used as a supposed monitor of Miocene shallow-water conditions is "Dentoglobigerina" altispira (e.g., Barrera et al., 1985; Prentice and Matthews, 1988; Corfield and Cartlidge, 1993; Norris et al., 1993; Prentice et al., 1993; Hodell and Vayavananda, 1994). "D." altispira consistently records negative $\delta^{18} \mathrm{O}$ and positive $\delta^{13} \mathrm{C}$ relative to other planktonic species. The advantage of this species is that individuals are large and common in lowlatitude sites (whereas G. ruber is often rare or absent). However, "D." altispira is an extinct species with no close living relative, so isotopic data provide virtually the only evidence for its life environment.

Figures $2 \mathrm{C}$ and $2 \mathrm{D}$ show the oxygen and carbon isotopic values given by " $D$." altispira in the early to middle Miocene, relative to the shallow- and deep-water reference taxa discussed above. The data are in line with those reported by other authors and broadly support a shallow-water habitat for "D." altispira. However, there is an apparent parallelism between the data for this species and the data for the presumed deep-dweller " $G$." venezuelana, particularly in $\delta^{13} \mathrm{C}$. Thus, it is possible that " $D$." altispira was a deep-dwelling species with a consistent vital effect of approximately $-1.0 \%$ in $\delta^{18} \mathrm{O}$ and $+1.5 \%$ in $\delta^{13} \mathrm{C}$. Further support for a possible deep-water habitat in " $D$." altispira is given by the low fluoride and magnesium concentrations found in the test reported by Opdyke and Pearson (this volume). Although not conclusive, these arguments at least raise a question mark over the use of "D." altispira as a shallow-water marker species.

\section{Fohsella Lineage}

Norris et al. (1993) and Hodell and Vayavananda (1994) have recently published detailed studies on the isotope systematics of the early to middle Miocene Fohsella (or "Globorotalia [Fohsella]") lineage at ODP Site 806 and DSDP Site 289, respectively. Both sites are in the western tropical Pacific. Norris et al. (1993) also provided data from DSDP Site 151 in the Caribbean. These authors proposed that the Fohsella lineage (which consists of the successive overlapping morphospecies $F$. peripheroronda, F. peripheroacuta, $F$. "praefohsi," and F. fohsi s.1.) was initially a shallow-water dweller but shifted its habitat preference into deeper water between 13.0 and $12.7 \mathrm{Ma}$. The supposed shift occurs at or slightly above the level of full "keel" development in the lineage (i.e., the first appearance datum of the F. fohsi morphospecies, which is used to recognize the base of Zone N12). The Fohsella lineage was preferentially sampled in this study to investigate whether a similar isotopic shift could be identified at Site 871 .

Figure 2E shows a detail of the oxygen isotope data, from below the base of Zone N8 to the hiatus level (which is thought to be high

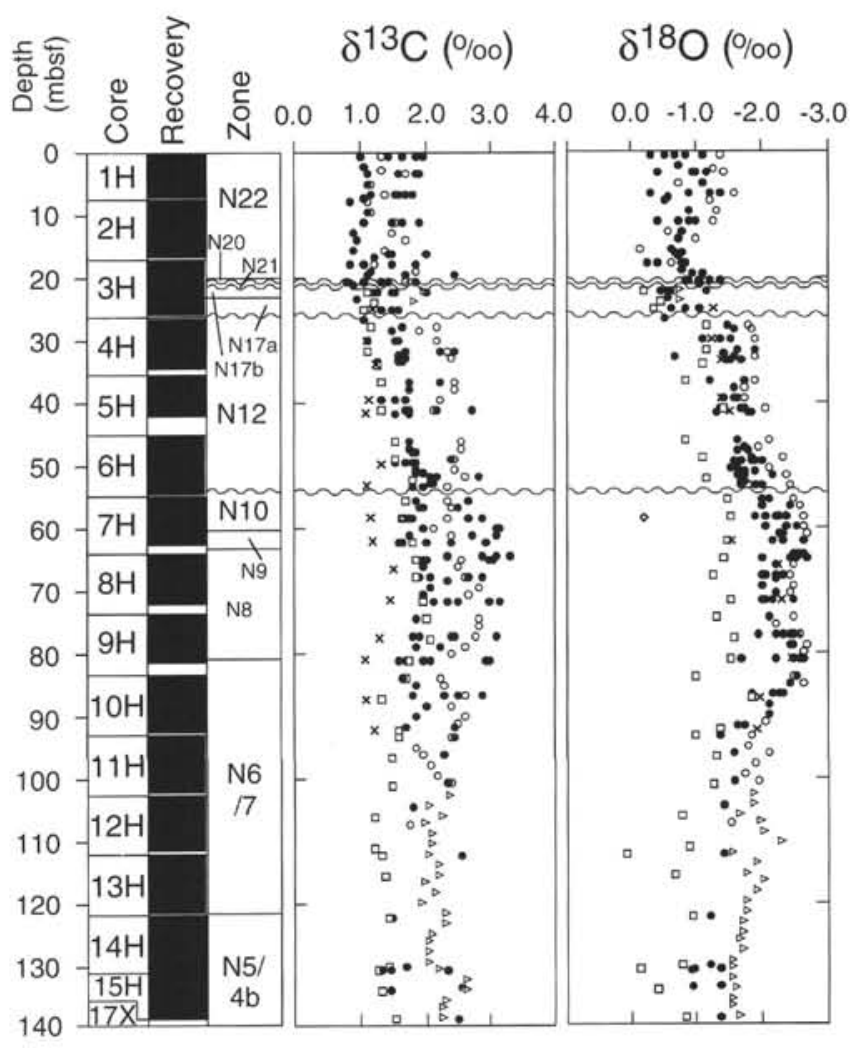

Figure 1. Carbon and oxygen isotope data from Hole 871A. The entire data set obtained in this study is plotted. Open circles = Globigerinoides ruber, open triangles = Globigerinoides trilobus s.1., open squares = "Globoquadrina" venezuelana, crosses $=$ Globigerinella praesiphonifera, diamond $=$ Clava torella bermudezi, and solid circles $=$ all other species. Planktonic foraminifer zonation is from Pearson (this volume).

in Zone N12 owing to the consistent presence of $F$. lobata and $F$. robusta). The Fohsella lineage is plotted as solid shapes and is compared to the reference taxa discussed above. The various morphospecies differentiated in the lineage are thought to belong to a single line of descent (Bolli, 1950, 1957, 1967; Blow and Banner, 1966; Kennett and Srinivasan, 1983).

The oxygen isotope data show relatively consistent isotopic values in Zones N8 through N10. Fohsella records values intermediate between the two reference taxa but closest to the shallow-dweller $G$. ruber. The absolute values are close to those of "D." altispira for this interval, as is also seen in the data of Norris et al. (1993) and Hodell and Vayavananda (1994). Directly above the minor unconformity at 54 mbsf, the first "keeled" specimens of Fohsella, attributed to $F$. fohsi, occur. These intergrade with $F$. "praefohsi" indicating a stratigraphic level toward the base of Zone N12. At this level, $\delta^{18} \mathrm{O}$ values change markedly to more positive values, as reported by Norris et al. (1993) and Hodell and Vayavananda (1994). However, this shift is also seen in the record of the shallow-water reference taxon G. ruber, which maintains a parallel $\delta^{18} \mathrm{O}$ record to Fohsella. Thus, the oxygen isotope data do not unequivocally support a deepening of the depth habitat of Fohsella at this site, as they could be explained equally by surface-water cooling and weakening of the thermocline, with Fohsella consistently occupying a relatively shallow habitat.

Norris et al. (1993) and Hodell and Vayavananda (1994) used "D." altispira as a surface-water reference taxon. If the G. ruber data were not available at this site, a deepening in the Fohsella habitat might be inferred because " $D$." altispira parallels Fohsella until within Zone $\mathrm{N} 12$, when it apparently becomes more negative and adopts values 
Table 1. Oxygen and carbon isotope data of multiple specimen samples of planktonic foraminifers, Hole 871A.

\begin{tabular}{|c|c|c|c|c|c|c|c|c|}
\hline $\begin{array}{l}\text { Core, section, } \\
\text { interval }(\mathrm{cm})\end{array}$ & $\begin{array}{l}\text { Depth } \\
\text { (mbsf) }\end{array}$ & Planktonic foraminifer species & $\delta^{18} \mathrm{O}$ & $\delta^{13} \mathrm{C}$ & $\begin{array}{l}\text { Core, section, } \\
\text { interval }(\mathrm{cm})\end{array}$ & $\begin{array}{l}\text { Depth } \\
\text { (mbsf) }\end{array}$ & Planktonic foraminifer species & $\delta^{18} \mathrm{O}$ \\
\hline 144-871A- & & & & & $5 \mathrm{H}-4,124-126$ & 41.74 & Globigerinella praesiphonifera & -1.51 \\
\hline $7 \mathrm{H}-3,59-61$ & 58.59 & Clavatorella bermudezi & -0.21 & 1.61 & $6 \mathrm{H}-3,124-126$ & 49.74 & Globigerinella praesiphonifera & -1.60 \\
\hline $4 \mathrm{H}-4.59-61$ & 31.59 & "Demoglobigerina" altispira & -1.87 & 2.20 & $6 \mathrm{H}-6,60-62$ & 53.60 & Globigerinella praesiphonifera & -1.87 \\
\hline $5 \mathrm{H}-1.59-61$ & 36.59 & "Dentoglobigerina" altispira & -1.71 & 2.21 & $7 \mathrm{H}-3,59-61$ & 58.59 & Globigerinella praesiphonifera & -2.30 \\
\hline $5 \mathrm{H}-4,59-61$ & 41.09 & "Dentoglobigerina" altispira & -1.68 & 2.16 & $\mathrm{H}-5,124-126$ & 62.24 & Globigerinella praesiphonifera & -1.53 \\
\hline $6 \mathrm{H}-3,60-62$ & 49.10 & "Dentoglobigerina" altispira & -1.81 & 2.38 & $8 \mathrm{H}-3,59-63$ & 66.59 & Globigerinella praesiphonifera & -2.21 \\
\hline $6 \mathrm{H}-6,60-62$ & 52.10 & "Dentoglobigerina" altispira & -2.11 & 2.81 & $8 \mathrm{H}-5,125-127$ & 71.75 & Globigerinella praesiphonifera & -2.27 \\
\hline $7 \mathrm{H}-3,59-61$ & 58.59 & "Dentoglobigerina" aitispira & -2.04 & 2.83 & $9 \mathrm{H}-3,59-6 \mathrm{I}$ & 77.59 & Globigerinella praesiphonifera & -2.53 \\
\hline $7 \mathrm{H}-5,124-126$ & 62.24 & "Dentoglobigerina" altispira & -2.31 & 2.87 & $9 \mathrm{H}-5,124-126$ & 81.24 & Globigerinella praesiphonifera & -2.45 \\
\hline $8 \mathrm{H}-3,59-63$ & 68.09 & "Dentoglobigerina" altispira & -2.29 & 2.86 & $10 \mathrm{H}-3,59-61$ & 87.09 & Globigerinella praesiphonifera & -1.95 \\
\hline $8 \mathrm{H}-5,125-127$ & 71.75 & "Dentoglobigerina" altispira & -2.14 & 3.13 & $10 \mathrm{H}-6,124-126$ & 92.24 & Globigerinella praesiphonifera & -1.92 \\
\hline $9 \mathrm{H}-3,59-61$ & 77.59 & "Dentoglobigerina" attispira & -2.31 & 3.07 & $\mathrm{IH}-1,59-61$ & 0.59 & Globigerinella siphonifera & -1.08 \\
\hline $9 \mathrm{H}-5,124-126$ & 81.24 & "Dentoglobigerina" altispira & -2.47 & 2.89 & $\mid \mathrm{H}-3,59-61$ & 3.59 & Globigerinella siphonifera & -1.14 \\
\hline $10 \mathrm{H}-3,59-61$ & 87.09 & "Dentoglobigerina" aitispira & -2.13 & 2.46 & IH-5, 59-61 & 6.59 & Globigerinella siphonifera & -1.35 \\
\hline $10 \mathrm{H}-6,124-126$ & 92.24 & "Dentoglobigerina" altispira & -1.68 & 2.39 & $2 \mathrm{H}-3,59-6 \mathrm{I}$ & 11.09 & Globigerinella siphonifera & -0.71 \\
\hline $11 \mathrm{H}-1,60-62$ & 93.60 & "Dentoglobigerina" altispira & -1.35 & 2.40 & $2 \mathrm{H}-6,124-126$ & 16.24 & Globigerinella siphonifera & -0.63 \\
\hline $11 \mathrm{H}-3,60-62$ & 96.60 & "Dentoglobigerina" altispira & -1.54 & 2.23 & $3 \mathrm{H}-3,60-62$ & 20.60 & Globigerinella siphonifera & -1.15 \\
\hline IIH-6, 60-62 & 101.10 & "Dentoglobigerina" altispira & -1.54 & 2.28 & $7 \mathrm{H}-4,59-6 \mathrm{I}$ & 60.09 & Globigerinoides bisphericus & -2.50 \\
\hline $12 \mathrm{H}-1,59-61$ & 104.49 & "Dentoglobigerina" altispira & -1.39 & 1.78 & $7 \mathrm{H}-5,59-61$ & 61.59 & Globigerinoides bisphericus & -2.30 \\
\hline $13 \mathrm{H}-1.58-60$ & 112.58 & "Dentoglobigerina" altispira & -1.37 & 2.52 & $7 \mathrm{H}-\mathrm{CC}$ & 64.50 & Globigerinoides bisphericus & -2.43 \\
\hline $14 \mathrm{H}-1,125-127$ & 122.75 & "Dentoglobigerina" altispira & -1.16 & 1.44 & $8 \mathrm{H}-1,59-63$ & 65.09 & Globigerinoides bisphericus & $-2,40$ \\
\hline $14 \mathrm{H}-6,125-127$ & 130.25 & "Dentoglobigerina" altispira & -1.17 & 1.69 & $3 \mathrm{H}-1,60-62$ & 17.60 & Globigerinoides fistulosus & -0.36 \\
\hline $15 \mathrm{H}-1.59-61$ & 131.09 & "Dentoglobigerina" altispira & -1.31 & 2.28 & $3 \mathrm{H}-2,123-125$ & 19.73 & Globigerinoides fistulosus & -1.07 \\
\hline $15 \mathrm{H}-3.59-6 \mathrm{I}$ & 134.09 & "Dentoglobigerina" altispira & -1.32 & 2.50 & $1 \mathrm{H}-1,59-61$ & 0.59 & Globigerinoides ruber & -1.32 \\
\hline $15 \mathrm{H}-6,59-61$ & 138.59 & "Dentoglobigerina" altispira & -1.35 & 2.49 & $\mid \mathrm{H}-2,59-61$ & 2.64 & Globigerinoides ruber & -1.21 \\
\hline $4 \mathrm{H}-\mathrm{I}, 124-126$ & 27.74 & Fohsella fohsi & -1.44 & 1.59 & $\mid \mathrm{H}-3,59-6 \mathrm{I}$ & 3.49 & Globigerinoides ruber & -1.41 \\
\hline $4 \mathrm{H}-2,59-61$ & 28.59 & Fohsella fohsi & -1.57 & 1.43 & $1 \mathrm{H}-4,59-61$ & 4.99 & Globigerinoides ruber & -0.71 \\
\hline $4 \mathrm{H}-2,124-126$ & 29.24 & Fohsella fohsi & -1.57 & 1.56 & $|H-5,59-6|$ & 6.49 & Globigerinoides ruber & -1.56 \\
\hline $4 \mathrm{H}-3,59-61$ & 30.09 & Fohsella fohsi & -1.50 & 1.50 & $2 \mathrm{H}-1,59-61$ & 8.09 & Globigerinoides ruber & -1.16 \\
\hline $4 \mathrm{H}-4,59-61$ & 31.59 & Fohsella fohsi & -1.59 & 1.64 & $2 \mathrm{H}-2,59-61$ & 9.59 & Globigerinoides ruber & -1.26 \\
\hline $4 \mathrm{H}-4,124-126$ & 32.24 & Fohsella fohsi & -1.37 & 1.54 & $2 \mathrm{H}-3,59-61$ & 11.09 & Globigerinoides ruber & -1.20 \\
\hline $4 \mathrm{H}-5,59-6 \mathrm{I}$ & 33.09 & Fohsella fohsi & -1.54 & 1.55 & $2 \mathrm{H}-4,59-61$ & 12.59 & Globigerinoides ruber & -0.52 \\
\hline $4 \mathrm{H}-5,124-126$ & 33.74 & Fohsella fohsi & -1.65 & 1.54 & $2 \mathrm{H}-5.59-6 \mathrm{I}$ & 14.09 & Globigerinoides ruber & -0.94 \\
\hline $5 \mathrm{H}-\mathrm{I}, 59-6 \mathrm{I}$ & 36.59 & Fohsella fohsi & -1.15 & 1.74 & $2 \mathrm{H}-6,59-61$ & 15.59 & Globigerinoides ruber & -0.13 \\
\hline $5 \mathrm{H}-1.124-126$ & 37.24 & Fohsella fohsi & -1.53 & 1.62 & $3 \mathrm{H}-1,60-62$ & 17.60 & Globigerinoides ruber & -0.60 \\
\hline $5 \mathrm{H}-2,59-6 \mathrm{I}$ & 38.09 & Fohsella fohsi & -1.57 & 1.72 & $3 \mathrm{H}-2,60-62$ & 19.10 & Globigerinoides ruber & -0.72 \\
\hline $5 \mathrm{H}-2,124-126$ & 38.74 & Fohsella fohsi & -1.65 & 1.68 & $3 \mathrm{H}-3,60-62$ & 20.60 & Globigerinoides ruber & -1.34 \\
\hline $5 \mathrm{H}-3,59-6 \mathrm{I}$ & 39.59 & Fohsella fohsi & -1.59 & 1.66 & $3 \mathrm{H}-6,60-62$ & 25.10 & Globigerinoides ruber & -1.79 \\
\hline $5 \mathrm{H}-4.59-61$ & 41.09 & Fohsella fohsi & -1.63 & 1.66 & $4 \mathrm{H}-1,126-8$ & 27.74 & Globigerinoides ruber & -1.74 \\
\hline $5 \mathrm{H}-4,124-126$ & 41.74 & Fohsella fohsi & -1.77 & 1.74 & $4 \mathrm{H}-2,59-6 \mathrm{I}$ & 28.59 & Globigerinoides ruber & -1.81 \\
\hline $6 \mathrm{H}-1,60-62$ & 46.10 & Fohsella fohsi & -1.62 & 1.71 & $4 \mathrm{H}-3,59-6 \mathrm{I}$ & 30.09 & Globigerinoides ruber & -1.86 \\
\hline $6 \mathrm{H}-1.124-126$ & 46.74 & Fohsella fohsi & -1.65 & 1.71 & $4 \mathrm{H}-4,59-6 \mathrm{I}$ & 31.59 & Globigerinoides ruber & -1.86 \\
\hline $6 \mathrm{H}-2,60-62$ & 47.60 & Fohsella fohsi & -1.70 & 1.70 & $4 \mathrm{H}-5,59-61$ & 33.09 & Globigerinoides ruber & -1.88 \\
\hline $6 \mathrm{H}-2,124-126$ & 48.24 & Fohsella fohsi & -1.76 & 1.83 & $5 \mathrm{H}-1,59-61$ & 36.59 & Globigerinoides ruber & -1.84 \\
\hline $6 \mathrm{H}-3,124-126$ & 49.74 & Fohsella foltsi & -1.81 & 1.83 & $5 \mathrm{H}-2,59-61$ & 38.09 & Globigerinoides ruber & -1.72 \\
\hline $6 \mathrm{H}-4,60-62$ & 50.60 & Fohsella fohsi & -1.49 & 1.85 & $5 \mathrm{H}-3,59-61$ & 39.59 & Globigerinoides nuber & -1.68 \\
\hline $6 \mathrm{H}-4.124-126$ & 51.24 & Fohsella fohsi & -1.69 & 1.94 & $5 \mathrm{H}-4.59-61$ & 41.09 & Globigerinoides ruber & -2.02 \\
\hline $6 \mathrm{H}-5,60-62$ & 52.10 & Fohsella fohsi & -1.59 & 2.14 & $6 \mathrm{H}-1,60-62$ & 46.10 & Globigerinoides ruber & -2.06 \\
\hline $6 \mathrm{H}-5,124-126$ & 52.74 & Fohsella fohsi & -1.64 & 2.07 & $6 \mathrm{H}-2.60-62$ & 47.60 & Globigerinoides ruber & -1.90 \\
\hline $6 \mathrm{H}-6,60-62$ & 53.60 & Fohsella peripheroacuta & -1.88 & 1.91 & $6 \mathrm{H}-3,60-62$ & 49.10 & Globigerinoides ruber & -2.27 \\
\hline $7 \mathrm{H}-1,59-6 \mathrm{I}$ & 55.59 & Fohsella peripheroacuta & -1.98 & 1.85 & $6 \mathrm{H}-4,60-62$ & 50.60 & Globigerinoides ruber & -2.06 \\
\hline $7 \mathrm{H}-2.59-61$ & 57.09 & Fohsella peripheroacuta & -1.95 & 1.93 & $6 \mathrm{H}-5,60-62$ & 52.10 & Globigerinoides ruber & -2.35 \\
\hline $7 \mathrm{H}-2,59-6 \mathrm{I}$ & 57.09 & Fohsella peripheroronda & -1.98 & 1.86 & $6 \mathrm{H}-6.60-62$ & 53.60 & Globigerinoides ruber & -2.38 \\
\hline $7 \mathrm{H}-3,59-6 \mathrm{I}$ & 58.59 & Fohsella peripheroronda & -2.26 & 1.72 & $7 \mathrm{H}-1.59-61$ & 55.59 & Globigerinoides ruber & -2.47 \\
\hline $7 \mathrm{H}-4,59-61$ & 60.09 & Fohsella peripheroronda & -2.02 & 1.92 & $7 \mathrm{H}-2,59-61$ & 57.09 & Globigerinoides ruber & -2.55 \\
\hline $7 \mathrm{H}-5,59-6 \mathrm{I}$ & 61.59 & Fohsella peripheroronda & -2.23 & 1.72 & $7 \mathrm{H}-3,59-6 \mathrm{I}$ & 58.59 & Globigerinoides nuber & -2.60 \\
\hline $8 \mathrm{H}-1,59-63$ & 65.09 & Fohsella peripheroronda & -2.00 & 1.91 & $7 \mathrm{H}-4,59-61$ & 60.09 & Globigerinoides ruber & -2.63 \\
\hline $8 \mathrm{H}-2.59-63$ & 66.59 & Fohsella peripheroronda & -2.19 & 1.91 & $7 \mathrm{H}-5,59-61$ & 61.59 & Globigerinoides ruber & -2.65 \\
\hline $8 \mathrm{H}-3.59-63$ & 68.09 & Fohsella peripheroronda & -2.20 & 1.89 & $8 \mathrm{H}-1.59-63$ & 65.09 & Globigerinoides ruber & -2.38 \\
\hline $8 \mathrm{H}-4,59-63$ & 69.59 & Fohsella peripheroronda & -1.96 & 2.03 & $8 \mathrm{H}-2,59-63$ & 66.59 & Globigerinoides ruber & -2.48 \\
\hline $8 \mathrm{H}-5.59-63$ & 71.09 & Fohsella peripheroronda & -2.18 & 1.91 & $8 \mathrm{H}-3,59-63$ & 68.09 & Globigerinoides ruber & -2.41 \\
\hline $9 \mathrm{H}-1,59-61$ & 74.59 & Fohsella peripheroronda & -2.07 & 1.81 & $8 \mathrm{H}-4,59-63$ & 69.59 & Globigerinoides ruber & -2.44 \\
\hline $9 \mathrm{H}-3,59-61$ & 77.59 & Fohsella peripheroronda & -2.39 & 1.77 & $8 \mathrm{H}-5,59-63$ & 71.09 & Globigerinoides ruber & -2.42 \\
\hline $9 \mathrm{H}-5,124-126$ & 81.24 & Fohsella peripheroronda & -2.43 & 1.57 & $9 \mathrm{H}-1.59-61$ & 74.59 & Globigerinoides ruber & -2.44 \\
\hline $6 \mathrm{H}-2,124-126$ & 48.24 & Fohsella "praefohsi" & -1.62 & 1.76 & $9 \mathrm{H}-2,59-6 \mathrm{I}$ & 76.09 & Globigerinoides ruber & -2.20 \\
\hline $6 \mathrm{H}-3,124-126$ & 49.74 & Fohsella "praefohsi" & -1.64 & 1.78 & $9 \mathrm{H}-3,59-61$ & 77.59 & Globigerinoides ruber & -2.54 \\
\hline $6 \mathrm{H}-4,60-62$ & 50.60 & Fohsella "praefohsi" & -1.58 & 1.84 & $9 \mathrm{H}-4,59-6 \mathrm{I}$ & 79.09 & Globigerinoides ruber & -2.65 \\
\hline $6 \mathrm{H}-4,124-126$ & 51.24 & Fohsella "praefohsi" & -1.89 & 1.83 & $9 \mathrm{H}-5,59-6 \mathrm{I}$ & 80.59 & Globigerinoides ruber & -2.59 \\
\hline $6 \mathrm{H}-5,60-62$ & 52.10 & Fohsella "praefohsi" & -1.68 & 2.04 & $10 \mathrm{H}-1,59-61$ & 84.09 & Globigerinoides ruber & -2.46 \\
\hline $6 \mathrm{H}-5,124-126$ & 52.74 & Fohsella "praefohsi" & -1.71 & 2.05 & $10 \mathrm{H}-2,59-61$ & 85.59 & Globigerinoides ruber & -2.61 \\
\hline $7 \mathrm{H}-5,124-126$ & 62.24 & Globigerinatella insueta & -2.61 & 2.35 & $10 \mathrm{H}-3,59-61$ & 87.09 & Globigerinoides ruber & -2.24 \\
\hline $7 \mathrm{H}-\mathrm{CC}$ & 64.50 & Globigerinatella insueta & -2.54 & 2.28 & $10 \mathrm{H}-4,59-61$ & 88.59 & Globigerinoides ruber & -2.07 \\
\hline $8 \mathrm{H}-1,59-61$ & 65.09 & Globigerinatella insueta & -2.50 & 2.01 & $10 \mathrm{H}-5,59-61$ & 90.09 & Globigerinoides ruber & -2.07 \\
\hline $8 \mathrm{H}-3,125-127$ & 68.75 & Globigerinatella insueta & -2.19 & 2.33 & $10 \mathrm{H}-6,59-61$ & 91.59 & Globigerinoides ruber & -2.02 \\
\hline $8 \mathrm{H}-5,125-127$ & 71.75 & Globigerinatella insueta & -2.05 & 2.33 & $11 \mathrm{H}-1,60-62$ & 93.60 & Globigerinoides ruber & -1.81 \\
\hline $9 \mathrm{H}-359-61$ & 77.59 & Globigerinatella insueta & -2.46 & 2.37 & $11 \mathrm{H}-2,60-62$ & 95.10 & Globigerinoides ruber & -1.77 \\
\hline $9 \mathrm{H}-4,59-61$ & 79.09 & Globigerinatella insueta & -2.45 & 2.20 & $11 \mathrm{H}-3,60-62$ & 96.60 & Globigerinoides ruber & -2.10 \\
\hline $9 \mathrm{H}-5,124-126$ & 81.24 & Globigerinatella insueta & -2.60 & 2.04 & $11 \mathrm{H}-4,60-62$ & 98.10 & Globigerinoides ruber & -1.87 \\
\hline $9 \mathrm{H}-4,59-61$ & 79.09 & Globigerinatella sp. & -2.40 & 1.80 & $11 \mathrm{H}-5,60-62$ & 99.60 & Globigerinoides ruber & -1.71 \\
\hline $9 \mathrm{H}-5,124-126$ & 81.24 & Globigerinatella sp. & -2.59 & 1,73 & $11 \mathrm{H}-6,60-62$ & 101.10 & Globigerinoides ruber & -1.90 \\
\hline $10 \mathrm{H}-1.59-61$ & 84.09 & Globigerinatella sp. & -2.51 & 1.61 & $12 \mathrm{H}-4,59-61$ & 107.59 & Globigerinoides ruber & -1.96 \\
\hline $10 \mathrm{H}-2,59-61$ & 85.59 & Globigerinatella sp. & -2.41 & 1.81 & $3 \mathrm{H}-4,60-62$ & 22.10 & Globigerinoides trilobus & -0.71 \\
\hline $\mathrm{IOH}-3,59-61$ & 87.09 & Globigerinatella sp. & -2.31 & 2.24 & $3 \mathrm{H}-5,60-62$ & 23.60 & Globigerinoides trilobus & -0.73 \\
\hline $10 \mathrm{H}-4,59-61$ & 88.59 & Globigerinatella sp. & -2.06 & 2.01 & $12 \mathrm{H}-1,59-61$ & 103.09 & Globigerinoides trilobus & -1.84 \\
\hline $10 \mathrm{H}-5,59-61$ & 90.09 & Globigerinatella sp. & -2.08 & 1.80 & $\mid 2 \mathrm{H}-2,59-61$ & 104.59 & Globigerinoides trilobus & -1.86 \\
\hline $3 \mathrm{H}-6,60-62$ & 25.10 & Globigerinella praesiphonifera & -1.26 & 1.18 & $12 \mathrm{H}-3,59-61$ & 106.09 & Globigerinoides trilobus & -1.61 \\
\hline $4 \mathrm{H}-3.59-61$ & 30.09 & Globigerinella praesiphonifera & -1.24 & 1.11 & $\mid 2 \mathrm{H}-4,59-61$ & 107.59 & Globigerinoides trilobus & -1.93 \\
\hline $4 \mathrm{H}-5,124-126$ & 33.74 & Globigerinella praesiphonifera & -1.37 & 1.24 & $12 \mathrm{H}-5,59-61$ & 109.09 & Globigerinoides trilobus & -1.97 \\
\hline $5 \mathrm{H}-3,59-6 \mathrm{I}$ & 39.59 & Globigerinella praesiphonifera & -1.38 & 1.14 & $12 \mathrm{H}-6,59-61$ & 110.59 & Globigerinoides trilobus & -2.27 \\
\hline
\end{tabular}


Table 1 (continued).

\begin{tabular}{|c|c|c|c|c|c|c|c|c|c|}
\hline $\begin{array}{l}\text { Core, section. } \\
\text { interval }(\mathrm{cm})\end{array}$ & $\begin{array}{l}\text { Depth } \\
\text { (mbsf) }\end{array}$ & Planktonic foraminifer species & $\delta^{18} \mathrm{O}$ & $\delta^{13} \mathrm{C}$ & $\begin{array}{l}\text { Core, section, } \\
\text { interval }(\mathrm{cm})\end{array}$ & $\begin{array}{l}\text { Depth } \\
\text { (mbsf) }\end{array}$ & Planktonic foraminifer species & $\delta^{18} \mathrm{O}$ & $\delta^{13} \mathrm{C}$ \\
\hline $13 \mathrm{H}-1.58-60$ & 112.58 & Globigerinoides trilobus. & -1.49 & 2.02 & IH-5, 59-61 & 6.59 & Globorotalia menardii & -1.15 & 1.51 \\
\hline $13 \mathrm{H}-2,58-60$ & 114.08 & Globigerinoides trilobus & -1.91 & 2.14 & $3 \mathrm{H}-6,60-62$ & 25.10 & Globorotalia menardii & -1.03 & 1.30 \\
\hline $13 \mathrm{H}-3,58-60$ & 115.58 & Globigerinoides trilobus & -1.72 & 2.14 & $3 \mathrm{H}-4,60-62$ & 22.10 & Globorotalia plesiotumida & -0.45 & 1.52 \\
\hline $13 \mathrm{H}-4,58-60$ & 117.08 & Globigerinoides trilobus & -2.02 & 1.93 & $3 \mathrm{H}-6,60-62$ & 25.10 & Globorotalia plesiotumida & -0.58 & 1.46 \\
\hline $13 \mathrm{H}-5,58-60$ & 118.58 & Globigerinoides trilobus & -1.87 & 2.09 & $4 \mathrm{H}-5,59-61$ & 33.09 & Globorotalia praemenardii & -0.64 & 1.64 \\
\hline $13 \mathrm{H}-6,58-60$ & 120.08 & Globigerinoides trilobus & -1.72 & 1.89 & IH-I, 59-61 & 0.59 & Globorotalia tumida & -0.25 & 1.85 \\
\hline $14 \mathrm{H}-1,60-62$ & 122.09 & Globigerinoides trilobus & -1.75 & 2.25 & $1 \mathrm{H}-5,59-61$ & 6.59 & Globorotalia tumida & -0.86 & 1.54 \\
\hline $14 \mathrm{H}-2,60-62$ & 123.60 & Globigerinoides trilobus & -1.65 & 2.25 & $\mid \mathrm{H}-3,59-61$ & 3.59 & Globorotalia tumida & -0.39 & 1.83 \\
\hline $14 \mathrm{H}-3,60-62$ & 125.10 & Globigerinoides trilobus & -1.67 & 2.08 & $2 \mathrm{H}-3,59-6 \mathrm{I}$ & 11.09 & Globorotalia tumida & -0.35 & 1.60 \\
\hline $14 \mathrm{H}-4,60-62$ & 126.60 & Globigerinoides trilobus & -1.61 & 1.99 & $3 \mathrm{H}-1,60-62$ & 17.60 & Globorotalia tumida & -0.22 & 1.45 \\
\hline $14 \mathrm{H}-5,60-62$ & 128.10 & Globigerinoides trilobus & -1.65 & 2.03 & $3 \mathrm{H}-2,123-125$ & 19.73 & Globorotalia tumida & -0.88 & 1.69 \\
\hline $14 \mathrm{H}-6,60-62$ & 129.60 & Globigerinoides trilobus & -1.50 & 2.01 & $3 \mathrm{H}-3,60-62$ & 20.60 & Globorotalia tumida & -0.79 & 1.40 \\
\hline $15 \mathrm{H}-1,59-61$ & 131.09 & Globigerinoides trilobus & -1.49 & 2.14 & $3 \mathrm{H}-5,60-62$ & 23.60 & Neogloboquadrina acostaensis & -0.53 & 0.90 \\
\hline $15 \mathrm{H}-2,59-6 \mathrm{I}$ & 132.59 & Globigerinoides trilobus & -1.50 & 2.59 & $4 \mathrm{H}-4,59-61$ & 31.59 & Orbulina suturalis & -1.89 & 2.42 \\
\hline $15 \mathrm{H}-3,59-6 \mathrm{I}$ & 134.09 & Globigerinoides trilobus & -1.56 & 2.59 & $5 \mathrm{H}-4,59-61$ & 41.09 & Orbulina suturalis & -1.72 & 2.68 \\
\hline $15 \mathrm{H}-4,59-61$ & 135.59 & Globigerinoides trilobus & -1.50 & 2.29 & $7 \mathrm{H}-1,59-61$ & 55.59 & Orbulina suturalis & -2.07 & 2.65 \\
\hline $15 \mathrm{H}-5,59-61$ & 137.09 & Globigerinoides trilobus & -1.50 & 2.20 & $7 \mathrm{H}-2,59-61$ & 57.09 & Orbulina suturalis & -2.39 & 2.47 \\
\hline $15 \mathrm{H}-6,59-6 \mathrm{I}$ & 138.59 & Globigerinoides trilobus & -1.50 & 2.22 & $7 \mathrm{H}-3,59-61$ & 58.59 & Orbulina suturalis & -2.35 & 2.61 \\
\hline $15 \mathrm{H}-1,59-6 \mathrm{I}$ & 131.09 & Globoquadrina binaiensis & -0.96 & 1.44 & $7 \mathrm{H}-4,59-61$ & 60.09 & Orbulina suturalis & -2.34 & 3.09 \\
\hline $15 \mathrm{H}-3,59-6 \mathrm{I}$ & 134.09 & Globoquadrina binaiensis & -0.93 & 1.31 & $7 \mathrm{H}-5,59-61$ & 61.59 & Orbulina suturalis & -2.31 & 2.67 \\
\hline $4 \mathrm{H}-3,59-61$ & 30.09 & Globoquadrina dehiscens & -1.31 & 1.06 & $7 \mathrm{H}-\mathrm{CC}$ & 64.50 & Orbulina suturalis & -2.49 & 3.03 \\
\hline $4 \mathrm{H}-5,124-126$ & 33.74 & Globoquadrina dehiscens & -1.46 & 1.21 & $\mid \mathrm{H}-1,59-61$ & 0.59 & Orbulina universa & -0.65 & 1.91 \\
\hline $5 \mathrm{H}-3,59-61$ & 39.59 & Globoquadrina dehiscens & -1.36 & 1.27 & $4 \mathrm{H}-3,59-61$ & 30.09 & Paragloborotalia mayeri & -1.07 & 1.58 \\
\hline $5 \mathrm{H}-4,124-126$ & 41.74 & Globoquadrina dehiscens & -1.29 & 1.73 & $4 \mathrm{H}-5,124-126$ & 33.74 & Paragloborotalia mayeri & -1.51 & 1.59 \\
\hline $6 \mathrm{H}-3,124-126$ & 49.74 & Globoquadrina dehiscens & -1.97 & 1.69 & $5 \mathrm{H}-3,59-61$ & 39.59 & Paragloborotalia mayeri & -1.53 & 1.52 \\
\hline $6 \mathrm{H}-6,60-62$ & 53.60 & Globoquadrina dehiscens & -1.66 & 2.33 & $5 \mathrm{H}-4,124-126$ & 41.74 & Paragloborotalia mayeri & -1.79 & 1.50 \\
\hline $7 \mathrm{H}-3,59-61$ & 58.59 & Globoquadrina dehiscens & -1.85 & 2.28 & $6 \mathrm{H}-3.124-126$ & 49.74 & Paragloborotalia mayeri & -1.55 & 1.77 \\
\hline $7 \mathrm{H}-5,124-126$ & 62.24 & Globoquadrina dehiscens & -2.14 & 1.56 & $6 \mathrm{H}-6,60-62$ & 53.60 & Paragloborotalia mayeri & -1.96 & 1.79 \\
\hline $8 \mathrm{H}-3,59-61$ & 68.09 & Globoquadrina dehiscens & $-2,04$ & 2.60 & $7 \mathrm{H}-3,59-61$ & 58.59 & Paragloborotalia mayeri & -2.17 & 1.76 \\
\hline $8 \mathrm{H}-5,125-127$ & 71.75 & Globoquadrina dehiscens & -1.95 & 2.47 & $7 \mathrm{H}-5,124-126$ & 62.24 & Paragloborotalia mayeri & -2.13 & 2.00 \\
\hline $9 \mathrm{H}-3.59-61$ & 77.59 & Globoquadrina dehiscens & -1.90 & 2.41 & $8 \mathrm{H}-3,59-61$ & 68.09 & Paragloborotalia mayeri & -1.98 & 2.02 \\
\hline $9 \mathrm{H}-5,124-126$ & 81.24 & Globoquadrina dehiscens & -1.63 & 1.94 & $8 \mathrm{H}-5,125-127$ & 71.75 & Paragloborotalia mayeri & -1.96 & 2.09 \\
\hline $15 \mathrm{H}-1,59-61$ & 131.09 & Globoquadrina praedehiscens & -0.98 & 1.38 & $9 \mathrm{H}-3,59-6 \mathrm{I}$ & 77.59 & Paragloborotalia mayeri & -2.19 & 1.89 \\
\hline $3 \mathrm{H}-4.60-62$ & 22.10 & Globoquadrina venezuelana & -0.17 & 1.08 & $9 \mathrm{H}-5,124-126$ & 81.24 & Paragloborotalia mayeri & -2.21 & 1.64 \\
\hline $3 \mathrm{H}-5,60-62$ & 23.60 & Globoquadrina venezuelana & -0.34 & 1.07 & $10 \mathrm{H}-3,59-61$ & 87.09 & Paragloborotalia mayeri & -1.82 & 1.77 \\
\hline $3 \mathrm{H}-6,60-62$ & 25.10 & Globoquadrina venezuelana & -0.43 & 1.18 & $10 \mathrm{H}-6,124-126$ & 92.24 & Paragloborotalia mayeri & -1.61 & 1.68 \\
\hline $4 \mathrm{H}-1,124-126$ & 27.74 & Globoquadrina venezuelana & -1.14 & 1.17 & 7H-CC & 64.50 & Praeorbulina glomerosa & -2.63 & 2.84 \\
\hline $4 \mathrm{H}-4,59-61$ & 31.59 & Globoquadrina venezuelana & -1.14 & 1.10 & $8 \mathrm{H}-1,59-63$ & 65.09 & Praeorbulina giomerosa & -2.67 & 2.93 \\
\hline $4 \mathrm{H}-5,124-126$ & 33.74 & Globoquadrina venezuelana & -1.08 & 1.28 & $8 \mathrm{H}-5,125-127$ & 71.75 & Praeorbulina glomerosa & -2.46 & 2.96 \\
\hline $5 \mathrm{H}-1,59-61$ & 36.59 & Globoquadrina venezuelana & -0.82 & 1.32 & $9 \mathrm{H}-3,59-6 \mathrm{I}$ & 77.59 & Praeorbulina glomerosa & -2.41 & 3.04 \\
\hline $5 \mathrm{H}-4,59-61$ & 41.09 & Globoquadrina venezuelana & -1.42 & 1.32 & $9 \mathrm{H}-5,124-126$ & 81.24 & Praeorbulina glomerosa & -2.54 & 2.93 \\
\hline $6 \mathrm{H}-1,60-62$ & 46.10 & Globoquadrina venezuelana & -0.82 & 1.52 & $10 \mathrm{H}-3,59-61$ & 87.09 & Praeorbulina glomerosa & -2.26 & 2.85 \\
\hline $6 \mathrm{H}-3,60-62$ & 49.10 & Globoquadrina venezuelana & -1.09 & 1.54 & $1 \mathrm{H}-1,59-61$ & 0.59 & Pulleniatina obliquiloculata & -0.82 & 0.98 \\
\hline $6 \mathrm{H}-6,60-62$ & 52.10 & Globoquadrina venezuelana & -1.13 & 1.77 & $\mid \mathrm{H}-1,59-61$ & 0.59 & Pulleniatina obliquiloculata & -1.23 & 1.29 \\
\hline $7 \mathrm{H}-1,59-61$ & 55.59 & Globoquadrina venezuelana & -1.48 & 1.71 & IH-2, 59-61 & 1.99 & Pulleniatina obliquiloculata & -0.67 & 1.02 \\
\hline $7 \mathrm{H}-3,59-61$ & 58.59 & Globoquadrina venezuelana & -1.52 & 1.61 & $1 \mathrm{H}-3,59-61$ & 3.59 & Pulleniatina obliquiloculata & -1.24 & 1.13 \\
\hline $7 \mathrm{H}-5,124-126$ & 62.24 & Globoquadrina venezuelana & -1.46 & 1.78 & $1 \mathrm{H}-3 ; 59-61$ & 3.49 & Pulleniatina obliquiloculata & -0.92 & 1.07 \\
\hline $8 \mathrm{H}-1,59-63$ & 65.09 & Globoquadrina venezuelana & -1.41 & 1.86 & $1 \mathrm{H}-4,59-61$ & 4.99 & Pulleniatina obliquiloculata & -1.09 & 1.08 \\
\hline $8 \mathrm{H}-3.59-63$ & 68.09 & Globoquadrina venezuelana & -1.23 & 1.83 & $1 \mathrm{H}-5,59-61$ & 6.49 & Pulleniatina obliquiloculata & -0.26 & 1.13 \\
\hline $8 \mathrm{H}-5,125-127$ & 71.75 & Globoquadrina venezuelana & -1.51 & 1.97 & $1 \mathrm{H}-5,59-6 \mathrm{I}$ & 6.59 & Pulleniatina obliquiloculata & -1.39 & 1.00 \\
\hline $9 \mathrm{H}-1,59-61$ & 74.59 & Globoquadrina venezuelana & -1.29 & 2.03 & IH-CC & 7.50 & Pulleniatina obliquiloculata & -0.53 & 1.00 \\
\hline $9 \mathrm{H}-3,59-61$ & 77.59 & Globoquadrina venezuelana & -1.55 & 2.08 & $2 \mathrm{H}-1,59-61$ & 8.09 & Pulleniatina obliquiloculata & -0.46 & 0.82 \\
\hline $9 \mathrm{H}-5,124-126$ & 81.24 & Globoquadrina venezuelana & -1.50 & 1.76 & $2 \mathrm{H}-2,59-61$ & 9.59 & Pulleniatina obliquiloculata & -0.87 & 1.10 \\
\hline $10 \mathrm{H}-1,59-61$ & 84.09 & Globoquadrina venezuelana & -0.98 & 1.66 & $2 \mathrm{H}-3,59-61$ & 11.09 & Pulleniatina obliquiloculata & -0.96 & 1.02 \\
\hline $10 \mathrm{H}-3,59-61$ & 87.09 & Globoquadrina venezuelana & -1.83 & 1.30 & $2 \mathrm{H}-3,59-6 \mathrm{I}$ & 11.09 & Pulleniatina obliquiloculata & -0.94 & 0.99 \\
\hline $10 \mathrm{H}-6.124-126$ & 92.24 & Globoquadrina venezuelana & -1.37 & 1.57 & $2 \mathrm{H}-4,59-61$ & 12.59 & Pulleniatina obliquiloculata & -0.74 & 0.84 \\
\hline $11 \mathrm{H}-1,60-62$ & 93.60 & Globoquadrina venezuelana & -0.97 & 1.60 & $2 \mathrm{H}-5,59-6 \mathrm{I}$ & 14,09 & Pulleniatina obliquiloculata & -0.67 & 0.94 \\
\hline $11 \mathrm{H}-3,60-62$ & 96.60 & Globoquadrina venezuelana & -1.28 & 1.49 & $2 \mathrm{H}-6,59-61$ & 15.59 & Pulleniatina obliquiloculata & -0.59 & 0.88 \\
\hline $11 \mathrm{H}-6,60-62$ & 101.10 & Globoquadrina venezuelana & -1.23 & 1.48 & $2 \mathrm{H}-6,124-126$ & 16.24 & Pulleniatina obliquiloculata & -0.66 & 1.39 \\
\hline $12 \mathrm{H}-3,59-61$ & 106.09 & Globoquadrina venezuelana & -0.79 & 1.23 & $2 \mathrm{H}-\mathrm{CC}$ & 17.00 & Pulleniatina obliquiloculata & -0.68 & 1.17 \\
\hline $12 \mathrm{H}-6,123-125$ & 111.23 & Globoquadrina venezuelana & -0.88 & 1.20 & $3 \mathrm{H}-1,60-62$ & 17.60 & Pulleniatina obliquiloculata & -0.77 & 0.83 \\
\hline $13 \mathrm{H}-1,58-60$ & 112.58 & Globoquadrina venezuelana & 0.07 & 1.33 & $3 \mathrm{H}-3,60-62$ & 20.60 & Pulleniatina obliquiloculata & -0.86 & 1.04 \\
\hline $13 \mathrm{H}-3,58-60$ & 115.58 & Globoquadrina venezuelana & -0.66 & 1.36 & $3 \mathrm{H}-1,123-5$ & 17.60 & Pulleniatina praecursor & -0.80 & 1.04 \\
\hline $14 \mathrm{H}-1,125-127$ & 122.75 & Globoquadrina venezuelana & -0.93 & 1.40 & $3 \mathrm{H}-2,60-62$ & 19.10 & Pulleniatina praecursor & -0.77 & 1.12 \\
\hline $14 \mathrm{H}-6.125-127$ & 130.25 & Globoquadrina venezuelana & -0.76 & 1.43 & $3 \mathrm{H}-2,123-5$ & 19.73 & Pulleniatina praecursor & -0.91 & 1.09 \\
\hline $15 \mathrm{H}-1,59-61$ & 131.09 & Globoquadrina venezuelana & -0.12 & 1.24 & $3 \mathrm{H}-3,60-62$ & 20.60 & Pulleniatina praecursor & -1.02 & 0.77 \\
\hline $15 \mathrm{H}-3,59-61$ & 134.09 & Globoquadrina venezuelana & -0.41 & 1.30 & $3 \mathrm{H}-3,123-5$ & 21.23 & Pulleniatina praecursor & -1.00 & 0.84 \\
\hline $15 \mathrm{H}-6,59-61$ & 138.59 & Globoquadrina venezuelana & -0.81 & 1.53 & $3 \mathrm{H}-4,60-62$ & 22.10 & Pulleniatina praecursor & -0.63 & 1.20 \\
\hline $5 \mathrm{H}-4,59-6 \mathrm{I}$ & 41.09 & Globorotalia archeomenardii & -1.33 & 1.70 & $3 \mathrm{H}-4,60-62$ & 22.10 & Pulleniatina primalis & -0.56 & 1.24 \\
\hline $6 \mathrm{H}-3,124-126$ & 49.74 & Globorotalia archeomenardii & -1.89 & 1.52 & $3 \mathrm{H}-4,123-5$ & 26.73 & Pulleniatina primalis & -0.48 & 1.01 \\
\hline $7 \mathrm{H}-3,59-61$ & 58.59 & Globorotalia archeomenardii & -2.26 & 1.68 & $1 \mathrm{H}-1,59-61$ & 0.59 & Sphaeroidinella dehiscens & -1.09 & 1.40 \\
\hline $7 \mathrm{H}-5,124-126$ & 62.24 & Globorotalia archeomenardii & -2.13 & 1.61 & $1 \mathrm{H}-5,59-61$ & 6.59 & Sphaeroidinella dehiscens & -1.35 & 1.79 \\
\hline $3 \mathrm{H}-4,60-62$ & 22.10 & Globorotalia limbata & -0.52 & 1.44 & $2 \mathrm{H}-3,59-61$ & 11.09 & Sphaeroidinella dehiscens & -0.84 & 1.89 \\
\hline $3 \mathrm{H}-6,60-62$ & 25.10 & Globorotalia limbata & -0.78 & 1.54 & $2 \mathrm{H}-6,124-126$ & 16.24 & Sphaeroidinella dehiscens & -0.74 & 1.99 \\
\hline $\mid \mathrm{H}-1,59-61$ & 0.59 & Globorotalia menardii & -0.50 & 1.63 & $3 \mathrm{H}-3,60-62$ & 20.60 & Sphaeroidinella dehiscens & -1.32 & 1.82 \\
\hline $1 \mathrm{H}-3,59-61$ & 3.59 & Globorotalia menardii & -0.96 & 1.58 & $3 \mathrm{H}-4,60-62$ & 22.10 & Sphaeroidinellopsis paenadehiscens & -1.10 & 1.98 \\
\hline
\end{tabular}

similar to G. ruber. However, only limited data for " $D$." altispira are currently available at this level.

The carbon isotopic data for the same interval are shown in Figure $2 \mathrm{~F}$. Fohsella has light carbon isotopes that would apparently indicate a deep habitat at the beginning of its range in Zone N8 (morphospecies Fohsella peripheroronda). Hoddell and Vayavananda (1994) and R.D. Norris (pers. comm., 1994) discovered a similar carbon isotope pattern at Sites 151,806, and 289. At all these sites, the earliest $F$. peripheroronda have a more negative $\delta^{13} \mathrm{C}$ than even " $G$." venezuelana, and later become more positive, but they do not approach the values given by the supposed surface dwellers. At face value these data would indicate a shallowing, not a deepening, during 

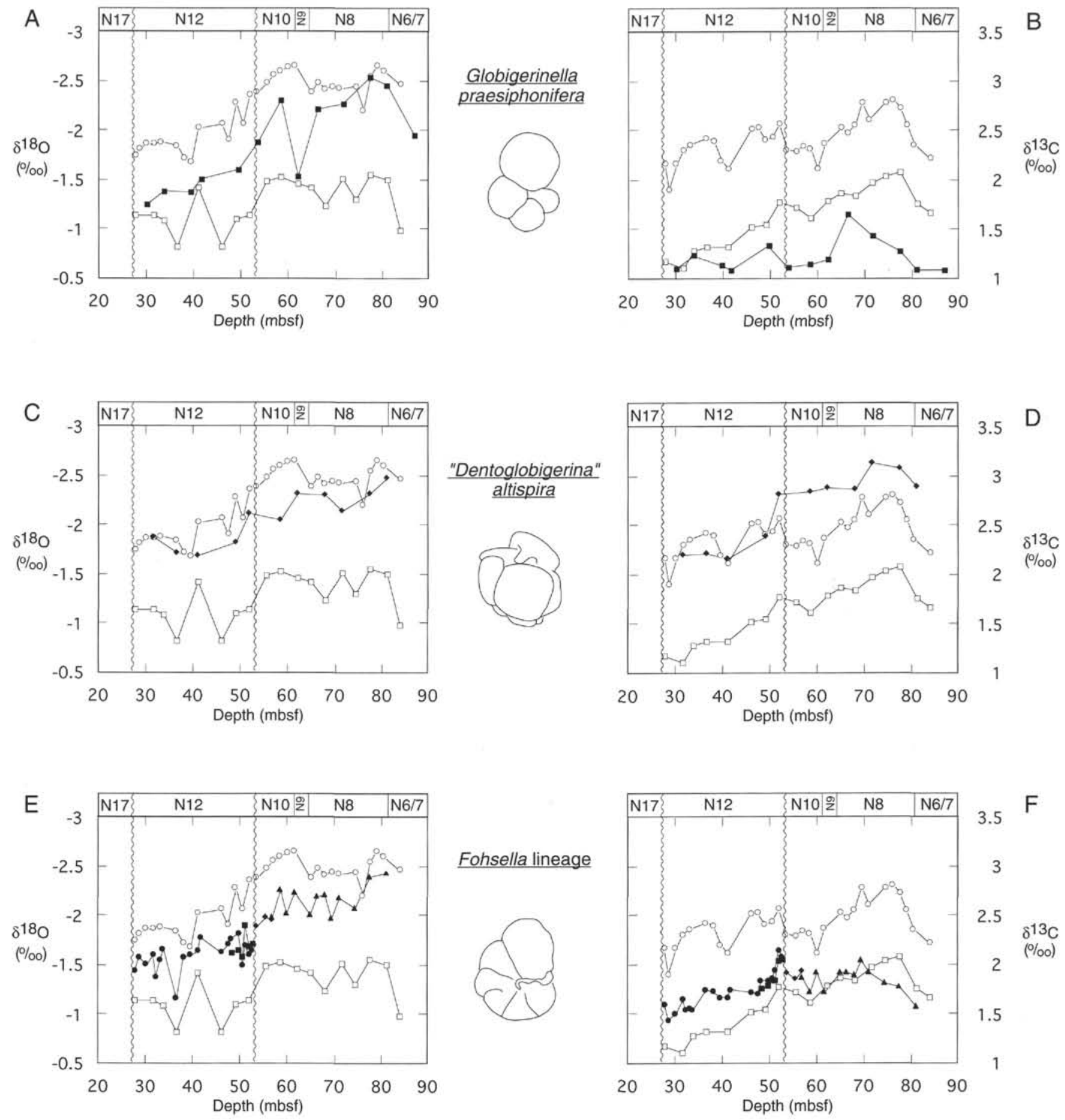

Figure 2. Middle Miocene isotope data, Hole 871A. Data for various species and species groups are shown relative to the records of Globigerinoides ruber (open circles) and "Globoquadrina" venezuelana (open squares). A, B. Solid squares = Globigerinella praesiphonifera. C, D. Solid diamonds = "Dentoglobigerina" altispira . E, F. Solid triangles = Fohsella peripheroacuta, solid diamonds = Fohsella peripheroronda, solid squares = Fohsella "praefohsi," and solid circles = Fohsella fohsi s.1. G, H. Solid triangles = Paragloborotalia mayeri. I, J. Solid triangles = Globoquadrina dehiscens. K, L. Solid squares $=$ Globigerinatella sp., and solid circles = Globigerinatella insueta $\mathbf{M}, \mathbf{N}$. Solid diamonds $=$ Globigerinoides bisphericus, solid squares $=$ Praeorbulina glomerosa, and solid circles $=$ Orbulina spp. O, P. Solid circles = Globorotalia archeomenardii, and solid squares = Globorotalia praemenardii. 

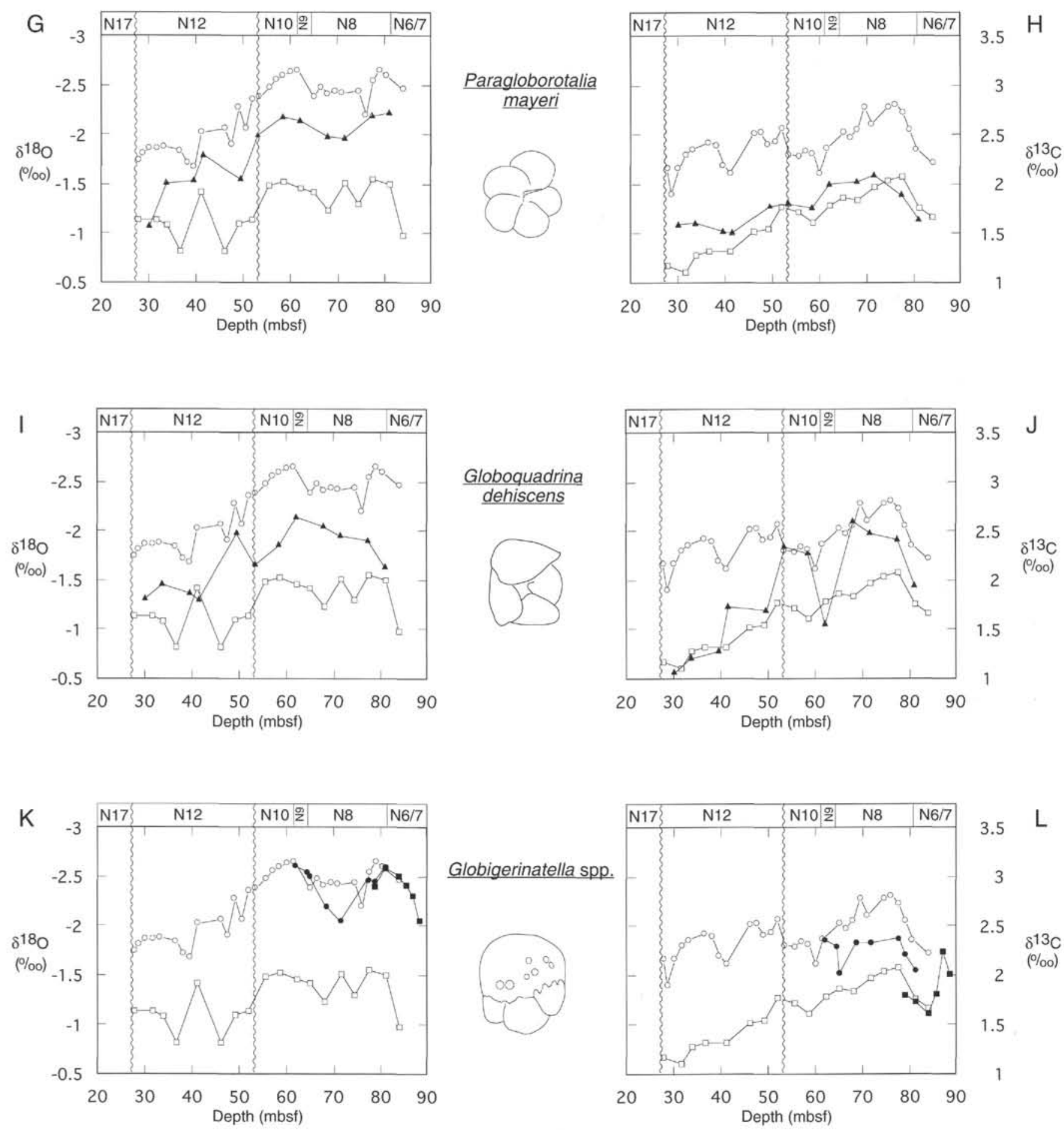

Figure 2 (continued).

the evolution of Fohsella. However, interpretations of carbon isotopes are generally less secure than oxygen owing to the greater possibility of disequilibrium fractionation. The strong parallelism between the carbon isotope records of $G$. ruber and Fohsella suggests that they calcified in similar environments but that Fohsella calcified out of equilibrium by approximately $-0.75 \%$ relative to $G$. ruber (making a total of $-1.25 \%$ relative to dissolved $\Sigma \mathrm{CO}_{2}$ ). If this interpretation is correct, the carbon isotope evidence do not support the hypothesis of a deepening in habitat during the evolution of Fohsella, because the $\delta^{13} \mathrm{C}$ offset between Fohsella and G. ruber is constant throughout its evolution, as is the case for $\delta^{18} \mathrm{O}$.

\section{Paragloborotalia mayeri}

The stable isotope record of Paragloborotalia mayeri (which includes "Paragloborotalia siakensis" of some authors) is shown in Figures $2 \mathrm{G}$ and $2 \mathrm{H}$ in comparison with the shallow- and deep-water reference taxa. This species shows isotopic values and trends almost 

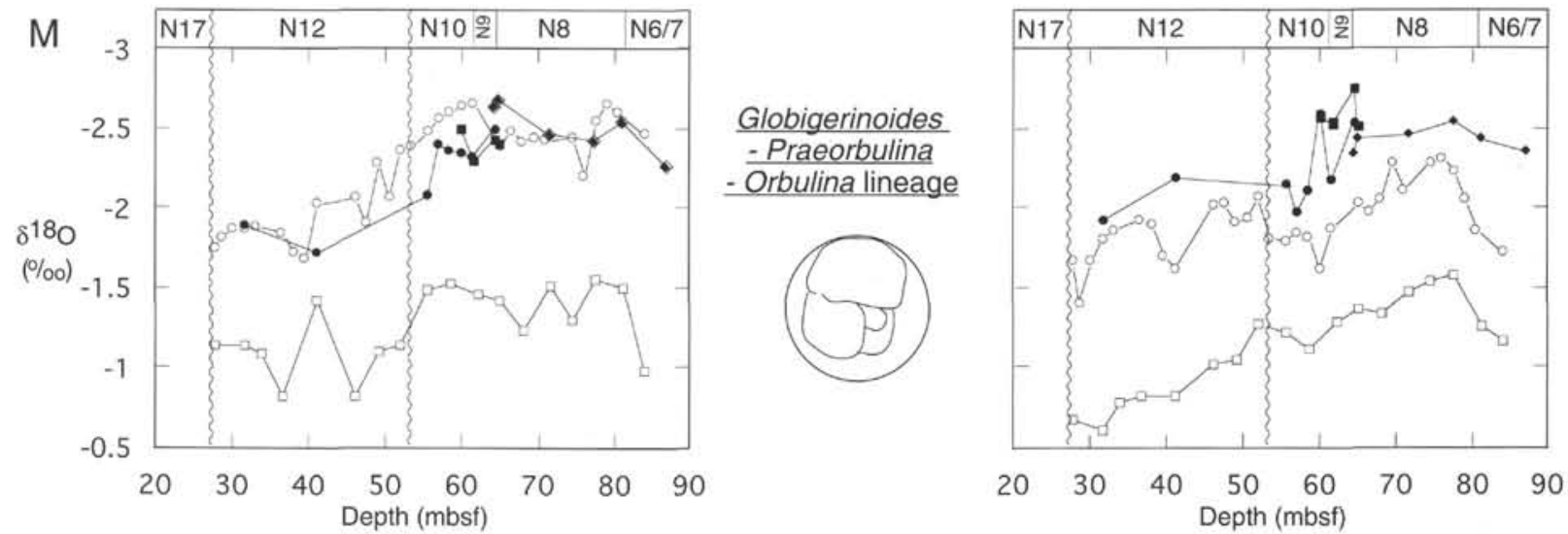

3.5

N

3

2.5

$\delta^{13} \mathrm{C}$

2

$(\%)$

1.5

1
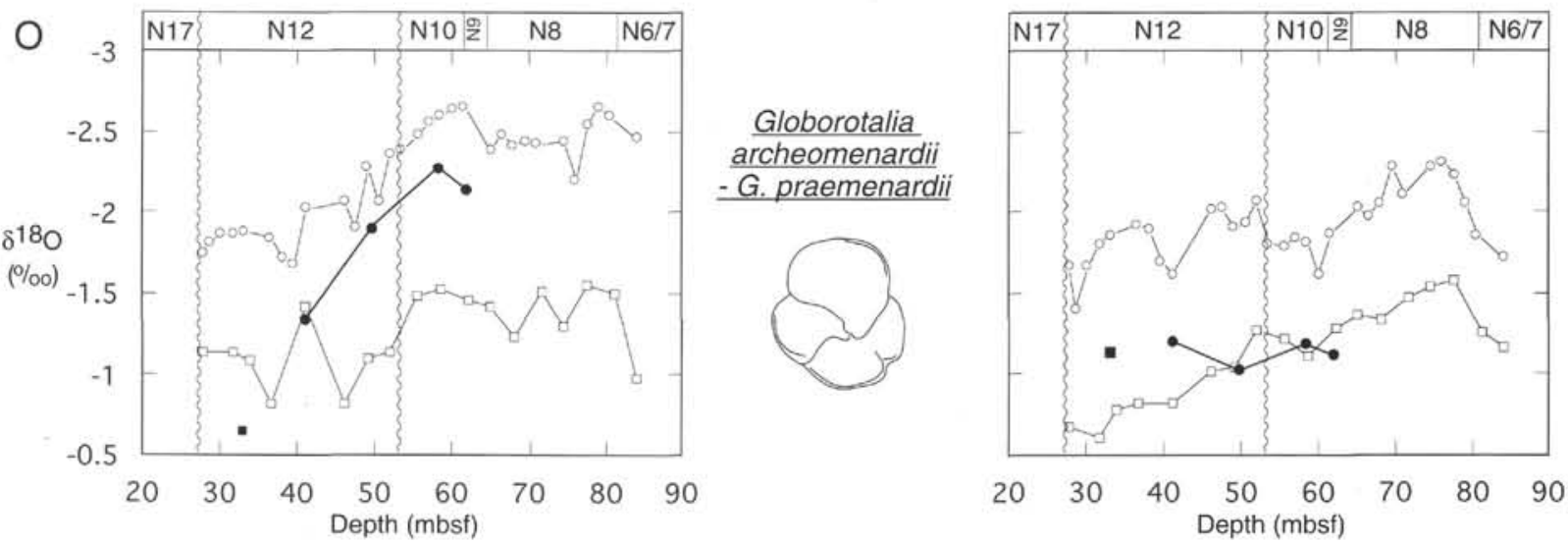

Globorotalia archeomenardii - G. praemenardii

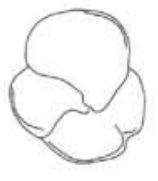

Figure 2 (continued).

indistinguishable from those given by Fohsella discussed above. Although relatively few data are available for this species, the isotopic trends appear to parallel the surface-dweller $G$. ruber with fairly constant offsets. Similar ratios were reported by Keller (1985) and Gasperi and Kennett (1992).

\section{Globoquadrina dehiscens}

Globoquadrina dehiscens was classified as an intermediate-water dweller by Keller (1985) on the basis of its oxygen isotopic ratios. In this study (Fig. 2I), the $\delta^{18} \mathrm{O}$ of $G$. dehiscens was generally recorded intermediate between $G$. ruber and " $G$." venezuelana and a little more positive than Fohsella. The $\delta^{13} \mathrm{C}$ is, however, erratic (Fig. 2J); the reasons for this are not clear, given the limited data.

\section{Globigerinatella spp.}

In this study, the genus Globigerinatella is split into two morphospecies, namely Globigerinatella sp. (forms lacking areal apertures) and Globigerinatella insueta (forms possessing areal apertures). A clear morphological trend was observed in the evolution of the lineage, as discussed in Pearson (this volume). Initial forms lack areal apertures and are attributable to Globigerinatella sp. The first specimens attributable to $G$. insueta occur at 100.24 mbsf but possess relatively few apertures and are subordinate to Globigerinatella $\mathrm{sp}$. until about $80 \mathrm{mbsf}$. Between $81 \mathrm{mbsf}$ and $77 \mathrm{mbsf}$, Globigerinatella $\mathrm{sp}$. becomes very rare and the number of apertures in $G$. insueta increases. A further increase in the mean number of apertures occurs near the extinction level of $G$. insueta between 65 and 62 mbsf.
Oxygen isotopic ratios in the Globigerinatella lineage are similar to those given by the shallow-water marker $G$. ruber (Fig. $2 \mathrm{~K}$ ). The $\delta^{13} \mathrm{C}$ is more erratic, however (Fig. 2L). The data, which are admittedly limited, point to a carbon isotope offset between those forms with and without areal apertures.

\section{Globigerinoides bisphericus-Praeorbulina-Orbulina Lineage}

The classic evolutionary transition from Globigerinoides to Orbulina, as first described by Blow (1956), is discussed for this site in Pearson (this volume). Isotopic results are shown in Figures $2 \mathrm{M}$ and $2 \mathrm{~N}$. The $\delta^{18} \mathrm{O}$ and $\delta^{13} \mathrm{C}$ data indicate that no great change of habitat accompanied the dramatic morphological changes. Both Orbulina and Globigerinoides trilobus, its ancestral form, are extant species and prefer shallow-water habitats with symbiotic associations. However, it is noted that the first appearance of Orbulina roughly coincides with a slight shift to more positive $\delta^{18} \mathrm{O}$. The consistent offset in $\delta^{13} \mathrm{C}$ of about $+0.5 \%$ for this lineage relative to $G$. ruber is interpreted as reflecting disequilibrium fractionation in $G$. ruber (see discussion under $G$. ruber above).

\section{Globorotalia archeomenardii}

Limited isotopic data for Globorotalia archeomenardii and a single sample of Globorotalia praemenardii are shown in Figures $2 \mathrm{O}$ and $2 P$. G. archeamenardii gives isotopic ratios similar to the other globorotaliids studied, Fohsella and Paragloborotalia. Hodell and Vayavananda (1994) hypothesized that the $G$. menardii lineage 
evolved from a deep-water habitat to an intermediate level at the time of the extinction of Fohsella, causing that extinction. These data suggest that at Site 871 the G. archeomenardii group already occupied a similar habitat to Fohsella at least 2 Ma before the extinction.

\section{Clavatorella bermudezi}

A single sample consisting of four large specimens of $C$. bermudez $i$ was analyzed. The $\delta^{18} \mathrm{O}$ is considerably more positive than any other middle Miocene species studied (see Fig. 1). The $\delta^{13} \mathrm{C}$ is also consistent with a deep-water habitat. In this respect, $C$. bermudez $i$ is comparable with the Eocene clavate form, Clavigerinella eocanica, which is thought to be a deep-water form (Pearson et al., 1993).

\section{PALEOCEANOGRAPHIC INTERPRETATION}

Published foraminifer isotopic data for much of the northwest and equatorial Pacific are relatively sparse owing to the limited number of suitable sites available. In this study, comparisons are made with other sites across the tropical Pacific, such as DSDP Sites 55 (Douglas and Savin, 1971), 77 (Savin et al., 1985), 158 (Keigwin, 1979), 289 (Shackleton, 1982; Savin et al., 1985), 292 (Savin et al., (1985), 448 (Savin et al., 1985), and 495 (Barrera et al., 1985). These data were synthesized and reviewed by Savin et al. (1985) and Barrera et al. (1985) and in other papers in Kennett (1985), whose synoptic maps provide the benchmark for Miocene paleoceanographic investigation. In addition, Gasperi and Kennett $(1992,1993 \mathrm{a})$ and Hodell and Vayavananda (1994) presented much new data from DSDP Site 289, Norris et al. (1993) presented data from ODP Site 806, and Prentice et al. (1993) presented data from Site 807.

As is well known, equilibrium oxygen isotopic ratios reflect both the temperature of calcification and the mean $\delta^{18} \mathrm{O}$ of seawater, which in turn is controlled by global ice-volume fluctuations. In the broadest terms, the oxygen isotopic differentiation between surface- (more negative) and deep-dwelling (more positive) planktonic foraminifers at Site 871 is in the region of $-1.0 \%$ throughout the time interval. However, intervals of reduced temperature differentiation may be present within Zone N6/7 (85-100 mbsf), Zone N12 (26-40 mbsf), and Zone N17 (23-26 mbsf). Whether these are "real" paleoceanographic effects, such as intervals with weakened thermoclines, or the result of stochastic variation arising from sampling error caused by individual variations cannot be determined with the present data. Also, because of this problem, no attempt is made to correlate using short-term oxygen isotopic excursions such as have been reported by Miller et al. (1991), and Woodruff and Savin (1991).

The broad temporal trend begins, in the early to middle Miocene, with a depletion of $\delta^{18} \mathrm{O}$ to a low in the interval of Zones N8 to N10, wherein surface-dwelling planktonic foraminifers record a $\delta^{18} \mathrm{O}$ of about $-2.5 \%$. This is interpreted as a climatic warming signal. Other Pacific sites show a surface warming through this interval (Savin et al., 1985; Corfield and Cartlidge, 1993) but in no recorded example is it of comparable magnitude. By Zone $\mathrm{N} 8, \delta^{18} \mathrm{O}$ values of both deepand shallow-dwelling planktonic foraminifers are more negative by as much as $-1.0 \%$ relative to other low-latitude Pacific sites such as Site 289 (Keller, 1985; Gasperi and Kennett, 1992). Data given in Opdyke and Pearson (this volume) show that Site 872 on Lo-En Guyot, which is just a few degrees of latitude to the north, does not show these anomalous paleotemperatures. The effect was persistent for a considerable period of time and suggests that Site 871 was located in a locally restricted warm current. The modern Equatorial Counter Current (ECC) may provide an analogy. Kennett et al. (1985) suggested that only a weak ECC existed in the early to middle Miocene. However, their study was based on inferences from limited data in the region.

In the stratigraphic interval of Zones N10 through N12, oxygen isotopic ratios of shallow-water species become considerably more positive, indicating a cooling trend. This appears to correspond to the benthic foraminifer $\delta^{18} \mathrm{O}$ increase between 14.9 and 12.9 Ma reported by Woodruff and Savin (1991). Typical values remain more negative than at neighboring sites, however, until above the major unconformity at 26 mbsf. These samples give $\delta^{18} \mathrm{O}$ values that are more positive still and comparable to those of the same age at other sites and indicate an end to the anomalously warm temperatures at Site 871 .

With the exception of Globigerinella praesiphonifera, which seems to display a strong carbon isotope vital effect, the $\delta^{13} \mathrm{C}$ differential between shallow- and deep-dwelling planktonic foraminifers is about 1.0 per mil throughout most of the Neogene at Site 871 (see Fig. 1 ). This suggests a relatively constant level of productivity in this region, which was always north of the paleoequator, but a detailed interpretation is not permitted because a wide variety of species are represented on Figure 1, each with an unknown vital effect.

The temporal trend in carbon isotopic ratios is in sympathy with those seen at other sites in benthic and planktonic foraminifers and primarily reflects variations in the global organic carbon balance. The interval of more positive $\delta^{13} \mathrm{C}$ between approximately 85 and $30 \mathrm{mbsf}$ corresponds approximately to the so-called "Monterey excursion" described by Vincent and Berger (1985), which is thought to represent a phase of accelerated organic carbon burial. No attempt is made to correlate by short-term variations in $\delta^{13} \mathrm{C}$ (cf. Woodruff and Savin, 1991) because of scatter caused by the relatively small number of individual foraminifers used in each sample.

\section{SUMMARY AND CONCLUSIONS}

The life environment of a variety of planktonic foraminifer species have been interpreted according to interspecies differences in their carbon and oxygen isotopic ratios. The interpretation of isotopic signatures is not simple, as data probably reflect both environmental conditions (principally depth in this case) and disequilibrium fractionation. Indeed, when several species are considered simultaneously, it is more difficult to make a straightforward interpretation of the data than if just one or two species are considered. Vital effects have to be invoked to explain reasonably the distribution of isotopic values between species. This is an important issue, because paleoceanographic interpretations can only be made if the magnitude of vital effects for each species is known or assumed.

The extent of disequilibrium fractionation in $\delta^{13} \mathrm{C}$ can be estimated in some cases by comparison with living individuals of the same species (as in Globigerinoides ruber) or in other cases by comparison with other species in the same fauna with similar $\delta^{18} \mathrm{O}$ (which assumes no vital effect in $\delta^{18} \mathrm{O}$ ). Parallelism of temporal trends between a species and a shallow- or deep-water reference taxon may also prove important in identifying suspect vital effects in both $\delta^{18} \mathrm{O}$ and $\delta^{13} \mathrm{C}$.

The modern shallow-dwelling form Globigerinoides ruber appears to have occupied a similar habitat since the early Miocene, with a small constant offset in $\delta^{13} \mathrm{C}$. This makes it a valuable near-surface marker species. However, because it is often rare in other sites, "Dentoglobigerina" altispira is a much more commonly used indicator of Miocene surface-water conditions, even though it is an extinct species with no living relative. In this study, an element of doubt is cast on the validity of using " $D$." altispira as a shallow-water marker because it does not follow the isotopic trend of $G$. ruber, but rather runs parallel the assumed deep-water form "Globoquadrina" venezuelana (to which it may be related). The trace element data of Opdyke and Pearson (this volume) also groups "D." altispira with inferred deep-dwelling forms.

Forms that parallel $G$. ruber include the Fohsella lineage, Paragloborotalia mayeri, and the Globigerinoides bisphericusPraeorbulina-Orbulina lineage. The former two examples show a further offset in $\delta^{13} \mathrm{C}$ relative to $G$. ruber, interpreted as a vital effect, whereas data from the Globigerinoides-Orbulina lineage are consistent with equilibrium surface-water conditions of both $\delta^{18} \mathrm{O}$ and $\delta^{13} \mathrm{C}$. 
No clear cases of evolutionary habitat change were found in the examples studied, despite the fact a number of fundamental morphological transitions were examined (e.g., Fohsella, Praeorbulina, Globigerinatella). A previously reported habitat change in Fohsella (Norris et al., 1993; Hodell and Vayavananda, 1994) is not supported in this study. Fohsella maintains a parallel record to G. ruber throughout until its stratigraphic range is truncated by hiatus.

\section{REFERENCES ${ }^{*}$}

Barrera, E., Keller, G., and Savin, S.M., 1985. Evolution of the Miocene ocean in the eastern North Pacific as inferred from oxygen and carbon isotopic ratios of foraminifera. In Kennett, J.P. (Ed.), The Miocene Ocean: Paleoceanography and Biogeography. Mem.-Geol. Soc. Am., 163:83-102.

Berger, W.H., 1969. Ecologic patterns of living planktonic foraminifera. Deep-Sea Res. Part A, 16:1-24.

Blow, W.H., 1956. Origin and evolution of the foraminiferal genus Orbulina d'Orbigny. Micropaleontology, 2:57-70.

Blow, W.H., and Banner, F.T., 1966. The morphology, taxonomy and biostratigraphy of Globorotalia barisanensis LeRoy, Globorotalia fohsi Cushman and Ellisor, and related taxa. Micropaleontology, 12:286-302.

Bolli, H.M., 1950. The direction of coiling in the evolution of some Globorotaliidae. Contrib. Cushman Found. Foraminiferal Res., 1:82-89.

, 1957. Planktonic foraminifera from the Oligocene-Miocene Cipero and Lengua formations of Trinidad, B.W.I. In Loeblich, A.R., Jr., Tappan, H., Beckmann, J.P., Bolli, H.M., Gallitelli, E.M., and Troelsen, J.C. (Eds.), Studies in Foraminifera. Bull.-U.S. Nat. Mus., 215:97-123.

1967. The subspecies of Globorotalia fohsi Cushman and Ellisor and the zones based on them. Micropaleontology, 13:502-512.

Bouvier-Soumagnac, Y.. Duplessey, J.C., and Bé, A.W.H., 1986. Isotopic composition of laboratory cultured planktonic foraminifers: implications for paleotemperature reconstructions. Oceanol. Acta, 9:519-522.

Corfield, R.M., and Cartlidge, J.E., 1991. Isotopic evidence for the depth stratification of fossil and Recent Globigerinina: a review. Hist. Biol., 5:37-63.

_ 1993. Oxygen and carbon isotope stratigraphy of the middle Miocene, Holes $805 \mathrm{~B}$ and 806B. In Berger, W.H., Kroenke, L.W., Mayer, L.A., et al., Proc. ODP, Sci. Results, 130: College Station, TX (Ocean Drilling Program), 307-322.

Douglas, R.G., and Savin, S.M., 1971. Isotopic analyses of planktonic foraminifers from the Cenozoic of the northwest Pacific, Leg 6. In Fischer, A.G., Heezen, B.C., et al., Init. Repts. DSDP, 6: Washington (U.S. Govt. Printing Office), 1123-1127.

1978. Oxygen isotopic evidence for the depth stratification of Tertiary and Cretaceous planktic Foraminifera. Mar. Micropaleontol., 3:175-196.

Erez, J., and Honjo, S., 1981. Comparison of isotopic composition of planktonic foraminifera in plankton tows, sediment traps and sediments. Palaeogeogr., Palaeoclimatol., Palaeoecol., 33:129-156.

Fairbanks, R.G., Sverdlove, M., Free, R., Wiebe, P.H., and Bé, A.W.H., 1982. Vertical distribution and isotopic fractionation of living planktonic foraminifera from the Panama Basin. Nature, 298:841-844.

Gasperi, J.T., and Kennett, J.P., 1992, Isotopic evidence for depth stratification and paleoecology of Miocene planktonic Foraminifera: western equatorial Pacific DSDP Site 289. In Tsuchi, R., and Ingle, J.C., Jr. (Eds.), Pacific Neogene: Environment, Evolution, and Events: Tokyo (Univ. of Tokyo Press), 117-147.

1993a. Miocene planktonic foraminifers at DSDP Site 289: depth stratification using isotopic differences. In Berger, W.H., Kroenke, L.W., Mayer, L.A., et al., Proc. ODP, Sci. Results, 130: College Station, TX (Ocean Drilling Program), 323-332.

Gasperi, J.T., and Kennett, J.P., 1993b. Vertical thermal structure evolution of Miocene surface waters: western Equatorial Pacific DSDP Site 289. Mar. Micropaleontol., 22:235-254.

Hemleben, C., Spindler, M., and Anderson, O.R., 1989. Modern Planktonic Foraminifera: Berlin (Springer-Verlag).

Hodell, D.A., and Vayavananda, A., 1994. Middle Miocene paleoceanography of the western equatorial Pacific (DSDP Site 289) and the evolution of Globorotalia (Fohsella). Mar. Micropaleontol., 22:279-310.

* Abbreviations for names of organizations and publications in ODP reference lists follow the style given in Chemical Abstracts Service Source Index (published by American Chemical Society).
Kahn, M.I., 1979. Non-equilibrium oxygen and carbon isotopic fractionation in tests of living planktonic foraminifera. Oceanol. Acta, 2:195-208.

Keigwin, L.D., Jr., 1979. Late Cenozoic stable isotope stratigraphy and paleoceanography of DSDP sites from the east equatorial and central North Pacific Ocean. Earth Planet. Sci. Lett., 45:361-382.

Keller, G., 1985. Depth stratification of planktonic foraminifers in the Miocene ocean. In Kennett, J.P. (Ed.), The Miocene Ocean: Paleoceanography and Biogeography. Mem.-Geol. Soc. Am., 163:177-196.

Kennett, J.P. (Ed.), 1985. The Miocene Ocean: Paleoceanography and Biogeography. Mem.-Geol. Soc. Am., 163.

Kennett, J.P., and Srinivasan, M.S., 1983. Neogene Planktonic Foraminifera: A Phylogenetic Atlas: Stroudsburg, PA (Hutchinson Ross).

Maynard Smith, J., 1988. Games, Sex and Evolution: Hemel Hempstead (Harvester Wheatsheaf)

Miller, K.G., Wright, J.D., and Fairbanks, R.G., 1991. Unlocking the Ice House: Oligocene-Miocene oxygen isotopes, eustasy, and margin erosion. J. Geophys. Res., 96:6829-6848.

Norris, R.D., Corfield, R.M., and Cartlidge, J.E., 1993. Evolution of depth ecology in the planktic foraminifera lineage Globorotalia (Fohsella). Geology, 21:975-978.

1994. Evolutionary ecology of Globorotalia (Globoconella) (planktic foraminifera). Mar. Micropaleontol., 23:121-145.

Pearson, P.N., Shackleton, N.J., and Hall, M.A., 1993. Stable isotope paleoecology of middle Eocene planktonic foraminifera and integrated isotope stratigraphy, DSDP Site 523, South Atlantic. I. Foraminiferal Res., 23:123-140.

Premoli Silva, I., Haggerty, J., Rack, F., et al., 1993. Proc. ODP, Init. Repts., 144: College Station, TX (Ocean Drilling Program).

Prentice, M.L., Friez, J.K., Simonds, G.G., and Matthews, R.K., 1993. Neogene trends in planktonic foraminifer $\delta^{18} \mathrm{O}$ from Site 807: implications for global ice volume and western equatorial Pacific sea-surface temperatures. In Berger, W.H., Kroenke, L.W., Mayer, L.A., et al., Proc. ODP, Sci. Results, 130: College Station, TX (Ocean Drilling Program), 281-305.

Prentice, M.L., and Matthews, R.K., 1988. Cenozoic ice-volume history: development of a composite oxygen isotope record. Geology, 16:963-966.

Ravelo, A.C., and Fairbanks, R.G., 1992. Oxygen isotopic composition of multiple species of planktonic foraminifera: recorders of the modern photic zone temperature gradient. Paleoceanography, 7:815-831.

Sautter, L., and Thunell, R., 1991. Seasonal variability in the $\delta^{18} \mathrm{O}$ and $\delta^{13} \mathrm{C}$ of planktonic foraminifera from an upwelling environment: sediment trap results from the San Pedro Basin, Southern California Bight. Paleoceanography, 6:307-334.

Savin, S.M., Abel, L., Barrera, E., Hodell, D.A., Keller, G., Kennett, J.P., Killingley, J.S., Murphy, M., and Vincent, E., 1985. The evolution of Miocene surface and near-surface marine temperatures: oxygen isotopic evidence. In Kennett, J.P. (Ed.), The Miocene Ocean: Paleoceanography and Biogeography. Mem.-Geol. Soc. Am., 163:49-82.

Shackleton, N.J., 1982. The deep-sea sediment record of climate variability. Prog. Oceanogr., 11:199-218.

Spero, H.J., 1992. Do planktic foraminifera accurately record shifts in the carbon isotopic composition of seawater \& $\mathrm{CO}_{2}$ ? Mar. Micropaleontol. 19:275-285.

Spero, H.J., and Williams, D.F., 1988. Extracting environmental information from planktonic foraminiferal $\delta^{13} \mathrm{C}$ data. Nature, 335:717-719.

Vincent, E., and Berger, W.H., 1985. Carbon dioxide and polar cooling in the Miocene: the Monterey Hypothesis. In Sundquist, E.T., and Broecker, W.S (Eds.), The Carbon Cycle and Atmospheric $\mathrm{CO}_{2}:$ Natural Variations Archean to Present. Geophys. Monogr., Am. Geophys. Union, 32:455468.

Vincent, E., Killingley, J.S., and Berger, W.H., 1985. Miocene oxygen and carbon isotope stratigraphy of the tropical Indian Ocean. In Kennett, J.P. (Ed.), The Miocene Ocean: Paleoceanography and Biogeography. Mem.-Geol. Soc. Am., 163:103-130.

Woodruff, F., and Savin, S.M., 1991. Mid-Miocene isotope stratigraphy in the deep-sea: high resolution correlations, paleoclimatic cycles, and sediment preservation. Paleoceanography, 6:755-806.

\footnotetext{
Date of initial receipt: 4 February 1994

Date of acceptance: 28 July 1994

Ms 144SR-054
} 